\title{
Photoperiod sensing system for timing of flowering in plants
}

\author{
Byoung-Doo Lee ${ }^{1, \#}$, Joon-Yung Cha ${ }^{2, \#}$, Mi Ri Kim ${ }^{2}$, Nam-Chon Paek ${ }^{1, *}$ E Woe-Yeon Kim ${ }^{2, *}$ \\ ${ }^{1}$ Department of Plant Science, Plant Genomics and Breeding Institute, Research Institute of Agriculture and Life Sciences, Seoul National \\ University, Seoul 08826, ${ }^{2}$ Division of Applied Life Science (BK21Plus), PMBBRC \& IALS, Gyeongsang National University, Jinju 52828, \\ Korea
}

\begin{abstract}
CONSTANS (CO) induces the expression of FLOWERING LOCUS $T(F T)$ in the photoperiodic pathway, and thereby regulates the seasonal timing of flowering. $\mathrm{CO}$ expression is induced and $\mathrm{CO}$ protein is stabilized by FLAVIN-BINDING KELCH REPEAT F-BOX PROTEIN 1 (FKF1) in the late afternoon, while $\mathrm{CO}$ is degraded by CONSTITUTIVE PHOTOMORPHOGENIC 1 (COP1) during the night. These regulatory cascades were thought to act independently. In our study, we investigated the relationship between FKF1 and COP1 in the regulation of $\mathrm{CO}$ stability in response to ambient light conditions. A genetic analysis revealed that FKF1 acts as a direct upstream negative regulator of COP1, in which cop1 mutation is epistatic to fkf1 mutation in the photoperiodic regulation of flowering. COP1 activity requires the formation of a hetero-tetramer with SUPPRESSOR OF PHYA-105 (SPA1), $\left[(\mathrm{COP} 1)_{2}(\mathrm{SPA} 1)_{2}\right]$. Light-activated FKF1 has an increased binding capacity for COP1, forming a FKF1-COP1 hetero-dimer, and inhibiting COP1 homo-dimerization at its coiled-coil (CC) domain. Mutations in the CC domain result in poor COP1 dimerization and misregulation of photoperiodic floral induction. We propose that FKF1 represses COP1 activity by inhibiting COP1 dimerization in the late aftemoon under long-day conditions, resulting in early flowering. [BMB Reports:
\end{abstract}

*Corresponding authors. Nam-Chon Paek, E-mail: ncpaek@snu.ac.kr; Woe-Yeon Kim, E-mail: kim1312@gnu.ac.kr

${ }^{\text {"TThe }}$ The authors contributed equally to this work.

https://doi.org/10.5483/BMBRep.2018.51.4.052

Received 10 March 2018

Keywords: CONSTANS, COP1, Dimerization, FKF1, Photoperiod, Timing of flowering

Abbreviations: CO, CONSTANS; COP1, CONSTITUTIVE PHOTOMORPHOGENIC 1; FKF1, FLAVIN-BINDING KELCH-REPEAT F-BOX PROTEIN 1; FMN, flavin mononucleotide; FT, FLOWERING LOCUS T; LAT, low ambient temperature; SPA1, SUPPRESSOR OF PHYA-105

Perspective to: Byoung-Doo Lee et al (2017), The F-box protein FKF1 inhibits dimerization of COP1 in the control of photoperiodic flowering, Nature Communications, 8:2259. DOI: 10.1038/s41467017-02476-2.
Perspective 2018; 51(4): 163-164]

In higher plants, flowering is the most important event for the preservation of the species. The process is tightly controlled by various signals such as light, water, temperature, biotic and abiotic stresses, and plant hormones. These signals activate the internal signal known as 'florigen', a hormone-like mobile molecule responsible for the onset of flowering, encoded by FLOWERING LOCUS T (FT) in Arabidopsis (Arabidopsis thaliana), Heading date $3 a$ (Hd3a) and Rice Flowering Locus $T$ (RFT) in Rice (Oryza sativa), TaFT1 in Wheat (Triticum aestivum), and HvFT1 in Barley (Hordeum vulgare). The flowering-time mechanism regulated by FT is known to be regulated by the photoperiodic, vernalization, autonomous, and hormonal pathways in Arabidopsis.

In the photoperiodic pathway, CONSTANS (CO) plays a central role in activating $F T$ expression for the induction of flowering in Arabidopsis. CO, a zinc-finger transcription factor, is regulated by the circadian clock and light conditions at the transcriptional and posttranslational levels, respectively. More specifically, $\mathrm{CO}$ transcription is controlled by the FLAVIN-BINDING KELCH REPEAT F-BOX PROTEIN 1 (FKF1)-GIGANTEA (GI)-CYCLING DOF FACTOR 1 (CDF1) regulatory module. Light-activated FKF1 interacts with GI and degrades CDF1, a key repressor of CO expression, to initiate $\mathrm{CO}$ transcription in a light-dependent manner. The $\mathrm{CO}$ protein is posttranslationally stabilized by FKF1 in the late afternoon, but degraded by CONSTITUTIVE PHOTOMORPHOGENIC 1 (COP1) during the night. In our study, we suggest that FKF1 directly represses COP1 to promote CO protein stability. In long-day (LD) conditions, light-activated FKF1 interacts with COP1 and inhibits its homo-dimerization, which is required for COP1 to form a hetero-tetramer $\left[(\mathrm{COP} 1)_{2}(\mathrm{SPA} 1)_{2}\right]$ complex that enables its function. In short-day (SD) conditions, FKF1 is mainly expressed in the dark, which results in inactive-FKF1 that cannot prevent COP1 homo-dimerization. Previous reports have shown that FKF1 and COP1 are independently involved in flowering via their regulation of $\mathrm{CO}$; however, we found that FKF1 and COP1 act in the same regulatory module, and that FKF1 inhibits COP1 activity to enhance CO protein stability, accelerating floral induction.

COP1 is a RING-type E3 ligase, which targets proteins 

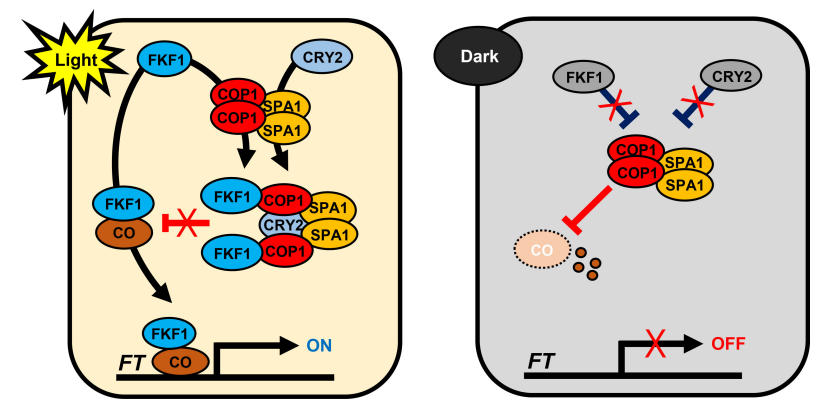

Diagram 1. Possible model of the FKF1-COP1-CO regulatory module in the photoperiod-dependent induction of flowering. The blue-light receptor FKF1 is highly expressed at ZT14 - ZT16, at which time the plants are exposed to light under LD conditions (16 $\mathrm{h}$ light/day), but are in the dark under SD (8 $\mathrm{h}$ light/day) conditions. Another blue-light receptor, CRY2, is stabilized in light, but quickly destabilized in darkness. In LD conditions, the light-activated blue-light receptors FKF1 and CRY2 interact with the COP1-SPA1 complexes, forming FKF1-COP1 and CRY2-SPA1 complexes. This might inhibit the formation of the COP1-SPA1 complexes in the late afternoon, resulting in the inactivation of COP1 or the destabilization of the COP1-SPA1 complex. Inhibition of COP1-SPA1 complex formation increases $\mathrm{CO}$ protein stability in the late afternoon, enabling it to interact with FKF1 and induce $F T$ transcription for floral induction. In SD conditions, both $C O$ and FKF1 are expressed in the dark, and CRY2 is destabilized in the dark. Thus, the blue-light receptors FKF1 and CRY2 are present in their inactive forms in darkness, and cannot destabilize COP1 complex, resulting in the rapid breakdown of $\mathrm{CO}$ by ubiquitin-dependent proteolysis.

involved in photo-morphogenesis [LONG HYPOCOTYL 5 (HY5) and HY5-HOMOLOG (HYH)] and flowering time (CO and $\mathrm{Gl}$ ) for $26 \mathrm{~S}$ proteasome-dependent degradation. COP1 is a light/dark-dependent nucleocytoplasmic partitioning protein that triggers the degradation of its target proteins under darkness. FKF1 is a component of the SKP1/CUL1/F-box (SCF)-type E3 ligase complex, and contains three distinct domains; LOV (Light, Oxygen, Voltage), F-box, and KELCH repeat domains. FKF1 acts as a blue-light receptor; the LOV domain binds to a flavin mononucleotide (FMN) chromophore to facilitate blue light sensing activity. FKF1 levels consistently peak at Zeitgeber time (ZT) 14 - ZT16, independent of the day length; thus, FKF1 is expressed in the daytime under LD, but during the night under SD conditions. In addition, the light activation of FKF1 protein only occurs under LD conditions. At LD dusk, COP1 exists in both the cytoplasm and the nucleus, but the $\mathrm{CO}$ protein is still stable due to the presence of active-FKF1. To recognize and transmit the light signal in plants, FKF1 acts as the main blue-light sensor of seasonal changes in photoperiod.

It remains unclear how FKF1 suppresses COP1 activity. Light-activated FKF1 interacts with the COP1 complex $\left[(\mathrm{COP} 1)_{2}(\mathrm{SPA} 1)_{2}\right]$, and inhibits COP1 homo-dimerization, but does not affect the COP1-SPA1 interaction. This regulatory activity may require the function of another blue-light receptor class, the CRYPTOCHROMEs (CRYs) as CRY1 and CRY2 interact with COP1 to inhibit its activity. Additionally, we found that other FKF1-family proteins, such as ZEITLUPE (ZTL) and LOV KELCH PROTEIN2 (LKP2), which have wellconserved LOV-domains that function as blue-light photoreceptors, also interact with COP1. Taken together, it is possible that blue-light receptors simultaneously inhibit COP1 activity by interacting with the COP1 complexes. To confer CO protein stability, FKF1 and CRY2 inhibit COP1 activity by simultaneously forming FKF1-COP1 and CRY2-SPA1 heterodimers. CRY1 and other ZTL proteins also interact with COP1 to regulate its other functions, such as hypocotyl elongation. The possibility that the blue-light receptors simultaneously inhibit COP1 activity remains to be examined.

COP1 is also involved in the regulation of floral induction in response to ambient temperatures, via a mechanism that involves the control of Gl protein stability. Under low ambient temperature $\left(\mathrm{LAT}, 16^{\circ} \mathrm{C}\right)$, the resulting delayed flowering is tightly controlled by the COP1 and GI proteins; as COP1 stability increases, and it facilitates degradation of GI protein. During the photoperiodic regulation of flowering, FKF1 forms a complex with $\mathrm{Gl}$; therefore, it is likely that FKF1 is also part of the regulatory machinery regulating flowering under LAT conditions, and further studies should investigate whether FKF1 senses not only light signals to control photoperiodic flowering, but also temperature fluctuations to regulate thermo-sensitive flowering.

A plant's survival depends on its ability to recognize fluctuating environmental conditions, and to select the optimal timing of flowering for its reproductive success. Our study suggests that FKF1 inhibits COP1 activity by repressing the homo-dimerization of COP1 in a light-dependent manner. Elucidating this machinery provides insight into the photoperiodic regulation of flowering. We also suggest that the blue-light receptors are involved in this regulatory pathway. Furthermore, we hypothesize that FKF1 influences the timing of flowering under LAT conditions. In this study, we elucidated the FKF1-COP1-CO regulatory pathway of flowering; however, these regulatory components might also be involved in other developmental processes, such as germination, photomorphogenesis, senescence, and defense mechanism. Further studies should examine the possibilities (Diagram 1).

\section{ACKNOWLEDGEMENTS}

This work was supported by Basic Science Research Program through the National Research Foundation of Korea (NRF) funded by the Ministry of Science, ICT and Future Planning (NRF-2017R1A2B3003310 to N.C.P. and NRF-2015R1D1A $1 \mathrm{~A} 01060604$ to B.D.L), and the Cooperative Research Program for Agriculture Science \& Technology Development (PJ010953042018 to W.Y.K.), Rural Development Administration, Republic of Korea. 